\title{
Social and Traditional Mainstream Media of Communication: Synergy and Variance Perspective
}

\section{Oberiri Destiny Apuke, Taraba State University, Nigeria}

\begin{abstract}
Social media are computer mediated tools that allow people to create, share or exchange information, ideas, pictures/videos in implicit communities and networks such as Facebook, Twitter, Baddo, Instagram among others. Whereas Traditional Media refer to those media that communicates identical messages in a one-way route to very large mass audiences, which are assumed to be homogeneous. Social media is at variance with Traditional media in production of media content; whereby social media content have now become more generally dispersed throughout the population, rather than restricted to media professionals, and there is more accessibility, quality and interactivity. Social media and the Traditional media share a relationship in advertisement, entertainment and news among others. The study postulates that although social media have come to stay, the traditional media will continue to strive in complimenting the social media.
\end{abstract}

Keywords: social media, traditional media, synergy, variance 


\section{Background of the Study}

The emergence of social media has increase interactivity among people, making them to be producers and consumers of information in a simultaneous manner. Social media such as Facebook, Twitter, 2go, etc. have brought tremendous improvement in the communication system, it has provided different entertainment functions which serve as a tool for social change. With the aid of the internet a user can form his/her own page, access to news online easily and upload business and advertisement online in order to get customers to purchase products. It is also an avenue to share or upload pictures of individual or events, groups can be formed to meet people with similar interests According to (Kim, kim and Kim, 2010): Social media are the emerging digital communication channels which create a user oriented information sharing ground where any people can generate or subscribe information content as both information provider and consumer. Although social media are powerful tools that has improved interactivity in communication, and reduced the utilization of traditional media, there are still some complimentary role it shares with traditional media in terms of news, advertisement, reaching large audiences among others. Whereas, variances traditional media shares with social media includes quality, frequency, usability, immediacy, and permanence (Social Media, 2013). Against this backdrop, the study seeks to explicate the variances and synergy social media shares with traditional mainstream media.

\section{Literature Review}

Literature reviews are embedded on documented sources by other authors related to the study. It reviews opinions by other scholars which strengthen the study, exploring the extent to which the topic has been investigated. To achieve this, the section is basically designed into:

(i) Concept of social media

(ii) Concept of traditional media

(iii) Variance Of Social Media To Traditional Media

(iv) Synergy Between Social And Traditional Media

(v) Reasons why Social Media cannot totally take away Traditional Media

\section{The Concept of Social Media}

Social media are computer mediated tools that allow people or companies to create, share exchange information, career interest, ideas, pictures/virtual communities and networks 
(Buettner, 2016). Through Social media, people can easily exchange valuable information which can improve their career interest, ideas etc. It also helps in sending pictorial illustrations of ideas. Kaplan and Haenlein (2010) see social media "as a group of internetbased applications that build on the ideological and technological foundations of web 2.0 and that allow the creation and exchange of user generated content". Kietzmann and Hermkens (2011) elaborates on this, stating that "social media depends on Mobile and Web-based technologies to create highly interactive platforms through which individuals and communities share, cocreate, discuss, and modify user-generated content. They introduce substantial land pervasive changes to communications between communities and individuals".

By implication, "social media is seen as the type of media that gives people medium to create, share, and/or exchange information and ideas in communities and networks. It is also seen as the ideological and technological exchange of user-generated content" (Social Media, 2013). This implies that they are the rising digital communication ground which create a user information sharing ground where people can be both information provider and consumer visavis through various forms such as; social blogs, weblogs, podcast, internet, social bookmarking etc.

\section{Types of Social Media}

There are several forms of social network as follow:

2go, Twitter, Facebook, WhatsApp, .... YouTube, Google+.

Facebook: this social network site is one of the most popular networking site which allows users to create their own profiles which gives ample opportunity for uploading of pictures, videos etc. Facebook also allows users to send and receive electronic messages via labtops, handsets and desktop computers as far as they are connected to the internet.

Twitter: twitter is one of the leading social sites used by people today. This site allows users to tweet shorts messages on their walls which attracts comments from other users. Users can follow others by tweeting back. 
Wikipedia: this is a free and open access online encyclopedia which allows people to create and upload documents that could be retrieved and used by others.

2go: this is a social media that is mostly found in mobile internet. It allows users to send and receive messages. Pictures, videos are also shared in this platform.

WhatsApp: this is a mobile social network that gives ample opportunity for users to interact freely with other users, allowing the sharing of pictures, videos, voice notes and other vital messages.

The functions of social media are enormous and very vital to the improvement of interactivity among people and this could range from Emailing, Messaging/chatting, Entertainment; Watching/Listening to music/clip, Online games, Advertisement blogs etc.

\section{Traditional Media of Communication}

Traditional media of communication could refer to those media that communicate uniform messages in a one-way process to large mass homogenous audience, all possessing much the same characteristics and interests. It deals with traditional broadcasting, like the terrestrial television channels such as (NTA, AIT, Channels TV and radio such as TSBS, BBC, UJ Fm etc). Asemah (2011) describes traditional media of communication as "channels or technological devices through which messages are conveyed to a large and heterogeneous audience". They are the vehicles that are used from conveying messages from a source to a large destination.

Some of the functions of traditional media range from surveillance, entertainment, cultural transmission, advertisement, agenda setting among others, etc.

\section{Variances between Social Media and Traditional Media of Mass Communication Some} of the properties that help describe the differences between social and Traditional media could be:

1. Expediency: social media are more easily accessible at a very low cost compared to the traditional media in which the production are typically government and/or corporate (privately 
owned) which requires consumers to sometimes pay for what they watch, but social media could even be accessed from a mobile device.

2. Usability: Traditional media requires special skills and training while social media production requires modest reinterpretation of existing skills which implies that anyone with access can operate the means of social media production.

3. Feedback: Feedback in Traditional media is delayed while social media's feedback is instant.

4. Permanence: Traditional media such as magazine article when printed and distributed cannot be altered; changes cannot be made to the same article but social media can be altered almost at every moment via comments or editing.

5. Interactivity: in social media users have an opportunity to or interact easily; creating their own material, customizing viewing to their own wishes, with much greater choice compared with the passive consumption and 'take it or leave it' features of the traditional media.

6. Dispersal. Social media unlike traditional media have become less centralized and more adapted to individual choices, with a huge growth of media products of all kinds, which have become a part of everyday life.

7. The production of media content has changed since the advent of social media unlike the traditional media that is reserved to media professionals for the media content and broadcasting, social media gives ample chances to individuals to make their own videos and post on the Internet.

\section{Synergy between Social and Traditional Media of Mass Communication}

Social media and the traditional media in some point share complimentary roles and functions as follows:

(i) One attribute shared by both social and Traditional media is the ability to reach small or large audiences; for example, facebook, twitter post or a television show may reach millions of people. This implies that both traditional and social media technologies provide are capable of reaching a large-scale audience.

(ii) Another complimentary role of social media and traditional media can be seen in facebook where online newspapers are posted; this combines traditional and social media to tell a story. 
(iii) Today, we now have online television where one can go and watch news and other related materials so it could be said that the traditional form of media are complimenting the new media in this aspect.

(iv) Advertisement is another aspect where social and traditional media's functions coincide. Both media's are medium of advertisement. The vanguard, the sun and other well known newspapers in Nigeria are projected in the social media where millions of adverts are placed for the consumption of the receiving public.

(v) Entertainment: Both social and the traditional media brings sense of humour and entertainment. There are programmes on television whereby social media addresses/links are given in order to make reply in such programmes. Instead of phone in programmes, it is the social media that is used for reply. For instance face book is a very popular medium used for feedback in many programmes on TV/Radio. This shows the complimentary role social media has on traditional media vis a vis.

(vi) News: The social media and the traditional media gives ample chance for audience to get news and information. For instance Newspaper houses have their website where they place daily news online. Hard copies are printed and distributed while the soft copies could be read online.

The aforementioned examples points to the fact that social media needs the traditional media vis a vis. They share complimentary roles of entertainment, advertising, news and information among others.

Although social media has come to stay with its benefits overshadowing the traditional media, there are still loop holes that give traditional media some edge over social media as follows:

\section{The Lack of Regulation}

The nature of freedom on social network sites such as facebook, twitter, 2 go has made it to lack regulation. Unlike the traditional media that is being regulated by different bodies in different countries, social media has no regulatory body which exposes it to bias, Internet crime (fraud) pornography, violence and racism among others because any can post anything at any time as long as he/she has the facility and the knowledge. 
The Decline Of Interpersonal Relationship: with the advent of Social Media isolation has increased, people lose the ability to communicate in the real world as they spend less quality time with family and friends, and have become more addicted to chatting and surfing the internet. Interpersonal relationship is on a decline with the advent of social media.

\section{The Digital Divide}

Not everyone has the means of obtaining social media gadgets such as laptops, handsets or even have knowledge on how to use the social media. This situation has brought about a digital divide between those who can and those who can't afford. This has created national and global inequalities, and a new digital. The aforementioned reasons still makes Traditional media (Radio, Television, Newspaper) thrive on.

\section{Summary and Conclusion}

From the foregoing, social media are computer mediated tools that allow people to create, share or exchange information, ideas, pictures/videos in virtual communities and networks such as Facebook, Twitter, Baddo, Instagram among others. Whereas Traditional Media refers to those media that communicated uniform messages in a one-way process to very large mass audiences, which are assumed to be homogeneous (all possessing much the same characteristics and interests). The social media is at variance with Traditional media in production of media content whereby social media content is now becoming more generally dispersed throughout the population, rather than restricted to media professionals. For example, people are now making their own videos and posting them on the Internet. Social Media and Traditional media share certain relation which has to do with the capability to reach small or large audiences; for example, either a blog post or a television show may reach no people or millions of people. This implies that both traditional and social media technologies provide scale and are capable of reaching a global audience.

\section{Recommendations}

Base on this study, the researcher proffer the following recommendations.

(1) Social Media platforms such as Facebook, twitter etc should have a regulatory body to avoid misinformation, pornography, racism in the globe. 
(2) Social media gadgets should be made cheap and readily available and training should be carried out to enable people get acquainted to the use of social media in order to reduce digital divide globally. 


\section{References}

Asemah, E. (2011). Principles and practice of Mass Communication. Jos: Great Future Press.

Buettner, R. (2016) "Getting a Job via Career oriented Social networking sites": The weakness of Ties. $49^{\text {th }}$ Annual International Conference on systems sciences. Kauai, Hawaii: IEEEE. https://doi.org/10.13140/RG. 2.1.3249.2241

Kaplan A. M., \& Haenlein M. (2010). Users of the world, unite! The challenges and opportunities of social media. Business Horizons, 53(1), 6-61.

Kietzmann, J. H., Hermkens, K., McCarthy, I. P., \& Silvestre, B. S. (2011). Social media? Get serious! Understanding the functional building blocks of social media. Business Horizons, 54(3), 241-251.

Kim, Y., Kim, M., \& Kim, K. (2010). Factors influencing the adoption of social media in perspective of information needs.

Social Media. (2013). Social media. In Wikipedia. Retrieved January 29, 2014 from http://en.wikipedia.org/wiki/Social_media 